\title{
(c)(1)(8) \\ Análisis comparativo del desarrollo motriz de niños de 4 y 5 años en contextos educativos urbanos y rurales.
}

\author{
Comparative analysis of the motor development of children of 4 and 5 \\ years old in urban and rural educational contexts
}

Jessica Paola Colcha Concha. ${ }^{1}$ \& Carolina Elizabeth San Lucas Solórzano. ${ }^{2}$

\begin{abstract}
.
Introduction. The adequate development of motor skills is an important factor for a correct adaptation of the child, this process is aimed at strengthening skills in order to lay the foundations so that subsequent learning that involves motor skills will be easier for the student. According to the guidelines of education in Ecuador, this factor must be standardized and respond to the mandatory curriculum established for initial education. However, the conditions of educational centers in urban and rural settings are influenced by different environmental, social and pedagogical factors. Objective. Establish if there are important differences in the acquisition of motor skills according to urban and rural learning environments. Methodology. For this, a study was carried out in 160 children from urban and rural schools, to which a test was applied to measure the level of gross and fine motor development, the results obtained were contrasted with the data obtained from a focus group made up of boys. family, teachers and technical specialists. Results. Statistical results indicate significant differences for gross motor skills, but there are no significant differences in fine motor skills. Conclusions. The findings found indicate that gross motor skills vary depending on the environments and based on the students' needs to adapt to them. Key Words: Gross Motor, Fine Motor, Urban Environment, Rural Environment, Motor Development
\end{abstract}

Keywords: Gross Motor, Fine Motor, Urban Environment, Rural Environment, Motor Development

\footnotetext{
${ }^{1}$ Pontificia Universidad Católica del Ecuador Sede Ambato, Maestría en Innovación Educativa, Ambato, Ecuador, jessica.p.colcha.c@ pucesa.edu.ec,ORCID 0000-0003-3547-5324

2 Pontificia Universidad Católica del Ecuador Sede Ambato, Maestría en Innovación Educativa, Ambato, Ecuador, karito_san@ hotmail.com Código ORCID 0000-0001-9791-3110
} 


\section{Resumen.}

Introducción. El desarrollo adecuado de la motricidad es un factor importante para una correcta adaptación del niño, este proceso, está orientado a fortalecer destrezas con el propósito de dejar sentadas las bases para que los posteriores aprendizajes que implican habilidades motoras le resulten al estudiante más fáciles. De acuerdo a los lineamientos de la educación en Ecuador, este factor debe estar estandarizado y responder al currículo obligatorio establecido para la educación inicial. Sin embargo, las condiciones de los centros educativos de los entornos urbano y rural se encuentran influidas por factores ambientales, sociales y pedagógicos diferentes. Objetivo. Establecer si existen diferencias importantes en la adquisición de la motricidad de acuerdo a los entornos de aprendizaje urbano y rural. Metodología. Para ello se realizado un estudio en 160 niños de escuelas urbanas y rurales, a los cuales se les aplico un test para medir el nivel de desarrollo motriz grueso y fino, los resultados obtenidos se contrastaron con los datos obtenidos de un grupo focal integrado por ladres de familia, docentes y técnicos especialistas. Resultados. Los resultados estadísticos indican diferencias significativas para la motricidad gruesa, pero no existen diferencias significativas en la motricidad fina. Conclusiones. Los hallazgos encontrados indican que la motricidad gruesa varía en función de los entornos y en base a las necesidades de los estudiantes para adaptarse a ellos.

Palabras claves: Motricidad Gruesa, Motricidad Fina, Entorno Urbano, Entorno Rural, Desarrollo motriz

\section{Introducción.}

En el contexto mundial, de acuerdo al informe de evaluación de la Educación (PISA, 2018) la educación en el Ecuador se encuentra ubicada entre las diez más bajas de Latinoamérica. Esto es el resultado de la deficiente administración de los procesos curriculares y las inadecuadas políticas educativas propuestas desde el gobierno central, en este contexto, la educación inicial juega un papel trascendental, ya que prepara a los estudiantes para los procesos posteriores, dando las destrezas necesarias para los aprendizajes posteriores de la lectura y la escritura, y con ellas los aprendizajes de las áreas básicas.

Para desarrollar estas capacidades, es importante que los niños en su primera infancia desplieguen las habilidades motoras suficientes para integrarse a los procesos educativos superiores de forma eficiente, este proceso depende de varios factores que involucran las esferas del desarrollo cognitivas, sociales y afectivas sobre las cuales los entornos influyen significativamente (Avendaño S, 2018). Si se considera, que los aprendizajes son el resultado de un cúmulo de experiencias que se vinculan entre sí y con el entorno para generar cambios de comportamiento, estos están influenciados además por elementos que intervienen en los procesos de aprendizaje como son las habilidades metodológicas de los docentes, los recursos pedagógicos y didácticos con los que cuenta 
la institución, el nivel de participación de la familia y la comunidad y sobre todo la predisposición anímica que el niño tenga para su desarrollo (Navas, 2018; Aceituno F \& Pina H, 2018) .

Al considerar la existencia de este complejo constructo de relaciones de aprendizaje, de herramientas de apoyo y condicionamientos físicas, cognitivos y afectivos, se ha propuesto en esta investigación analizar de qué manera se presentan estos factores en los dos entornos característicos de la realidad socio cultural ecuatoriana, esto es el sector urbano y el sector rural, que podrían estar influyendo de alguna manera en el desarrollo de las habilidades motoras de los niños, ya que se configuran como espacios en los cuales se desarrollan las destrezas en función de necesidades emergentes que los párvulos tienen para adaptarse al medio.

Los infantes necesitan aprender a moverse y usar sus cuerpos para realizar diversas tareas, proceso más conocido como desarrollo motor. Inicialmente, los movimientos de los niños son simplemente reflejos incontrolados con los que nacen, con el tiempo, aprenden a mover las partes de su cuerpo voluntariamente para realizar movimientos motores gruesos (grandes) y finos (pequeños). En general, los niños comienzan a desarrollar habilidades motoras desde el centro del cuerpo hacia afuera y desde la cabeza hacia las extremidades inferiores, controlan su cabeza y cuello antes de aprender a maniobrar sus brazos; aprenden a maniobrar sus brazos antes de aprender a manipular sus dedos, aprenden a mover el torso antes de mover los brazos y las piernas (Navas, 2018).

El desarrollo motor de un niño se divide en dos categorías: motor fino y motor grueso. Las habilidades motoras finas se refieren a pequeños movimientos en las manos, muñecas, dedos, pies, dedos de los pies, labios y lengua. Las habilidades motoras gruesas implican el desarrollo motor de los músculos que permiten a los bebés levantar la cabeza, sentarse, gatear y finalmente, caminar, correr y saltar (Avendaño S, 2018).

El desarrollo típico de las habilidades motoras sigue una secuencia predecible. Comienza desde el interior del cuerpo, incluida la cabeza, el cuello, los brazos, las piernas, y luego se mueve hacia el exterior del cuerpo, como las manos, los pies, los dedos de las manos y pies. El desarrollo motor es importante a lo largo de la vida temprana de un niño, porque el desarrollo físico está vinculado a otras áreas de desarrollo. Por ejemplo, si un niño puede gatear o caminar (habilidades motoras gruesas), puede explorar más fácilmente su entorno físico, lo que afecta el desarrollo cognitivo. El desarrollo social y emocional progresa cuando un niño puede hablar, comer y beber (habilidades motoras finas) (Pacheco, 2019).

Por su parte, Castillo (2018), propuso una conexión bidireccional entre competencias motoras y desarrollo cognitivo, asegurando que las competencias motoras influyen en la motivación y el compromiso de los niños en la actividad física y el desarrollo de aprendizajes, por lo tanto, la disminución de competencias motoras en los niños puede deberse a una baja de la actividad física o la calidad de los entornos en los que se desarrolla sus aprendizajes y su vida cotidiana. 
En este contexto, Zula y Comeca (2019), creadores de la teoría de la adaptabilidad, se refieren a las propiedades funcionalmente significativas del medio ambiente; describen la variedad de espacios que ofrece un entorno y como se puede utilizar para múltiples propósitos, de acuerdo a estos autores se define la adaptabilidad como una combinación de propiedades físicas del entorno que se ajustan a las acciones y los sistemas motores de los individuos.

La teoría de la adaptabilidad también ha sido aplicada a los contextos de aprendizaje motor de los niños pequeños, para explicar la actividad física, en términos de juego al aire libre, movilidad independiente y la forma de relacionarse con el entorno, se ha demostrado que los niños encuentran los entornos al aire libre estimulantes y motivadores, espacios amplios brindan posibilidades para jugar y correr, también son cruciales para practicar las habilidades de control de objetos es decir el desarrollo de la motricidad fina.

Arichabala (2019) sugirió que las habilidades motrices mejoradas proporcionan experiencias sociales y cognitivas más numerosas y variables y apoyan el desarrollo de tales experiencias durante la infancia. Después de la infancia, las habilidades motrices bien desarrollas pueden ser responsables de las capacidades para mantener y promover dicho desarrollo. Durante la primera infancia, la manipulación de diferentes objetos abre nuevas oportunidades para la exploración visual, manual y oral. Además de las habilidades motrices gruesas y finas, también la coordinación y el equilibrio son esenciales para el desarrollo de las capacidades motrices de los niños.

Todas las habilidades antes mencionadas sirven para desarrollar las capacidades motrices de los niños, tanto en intensidades de actividad física. Sin embargo, como se mencionó anteriormente, para practicar, por ejemplo, caminar o correr, es necesario contar con diferentes posibilidades además de escalar, lanzar o mantener el equilibrio. Esto demuestra que el entorno y sus posibilidades pueden contribuir al desarrollo motor y la actividad física (Silva Martínez, 2018).

El desarrollo motor no solo influye en el nivel de actividad física, sino que también está relacionado con las funciones cognitivas. Recientemente, la capacidad motriz se ha asociado con funciones cognitivas, como las funciones ejecutivas, la atención y la memoria de trabajo y la velocidad de procesamiento de la información, así como el desarrollo del lenguaje y la lectura. (Aceituno F \& Pina H, 2018).

Por lo tanto, para apoyar las funciones cognitivas, el entorno tiene un papel importante al proporcionar posibilidades para practicar las habilidades motoras. Por otro lado, para el aprendizaje motor, el momento perfecto para que el niño aprenda habilidades es esencial, por lo tanto, se reconoce como una zona de aprendizaje próximo. Cuando un niño tiene los requisitos previos para aprender una nueva habilidad, pero aún no puede hacerlo solo, el entorno u otras personas (compañeros o adultos) pueden apoyar y reforzar este nivel apropiado (Salazar C \& Jiménez D, 2020). 


\section{Desarrollo motriz}

\section{Desarrollo Motor}

La evidencia convergente de muchos campos de investigación diferentes sugiere que los movimientos humanos se organizan como acciones y no como reacciones. Sin embargo, todavía se define como la misma acción si el objetivo sigue siendo el mismo. Por tanto, el estado objetivo ya está representado cuando se planifican las acciones (Pacheco, 2019). $\mathrm{Al}$ ejecutar acciones u observar a otra persona realizarlas, los individuos fijan metas y sub-metas de los movimientos. Sin embargo, esto solo se hace si una acción está implícita al mostrar los mismos movimientos sin el contexto de un agente, los sujetos fijaron el movimiento en lugar de los objetivos. Otros estudios recientes de control motor también demuestran la estrecha relación entre percepción y acción (Reboiras, Cancela M, \& Ayán, 2015, p. 138).

\section{Factores del desarrollo motor}

La percepción y la planificación de los movimientos de los niños en la primera infancia parecen seguir principios similares a de los adultos. Los niños pequeños imitan el propósito de las acciones en lugar de su forma exacta y la tendencia a imitar las acciones depende de lo interesantes que sean sus efectos o resultados (Roque, et al, p. 68). Además, al observar las acciones realizadas por otros, los niños prestan más atención al propósito de los movimientos que a su forma exacta.

Un enfoque de acción para el desarrollo motor tiene varias implicaciones importantes. Primero, le da una importancia central a la planificación y predicción de movimientos. Las acciones están dirigidas al futuro y deben predecir lo que sucederá a continuación. Dicho control prospectivo se basa en el conocimiento de las reglas y regularidades que gobiernan los eventos en el mundo y en las habilidades para extraer información orientada al futuro de los sentidos (Garófano V \& Guirado C, 2017, p. 96).

En segundo lugar, un enfoque de acción enfatiza los factores motivacionales en el desarrollo motor. Estos factores pueden estar relacionados a los estímulos de los entornos es decir a los contextos urbanos y rurales. Un enfoque de acción enfatiza la guía perceptiva de los movimientos en lugar de la adquisición de programas motores. Esto hace referencia a los elementos que están dentro de cada contexto educativo y los caracterizan y diferencian unos de otros (Roque I, et al, p. 69).

Los sistemas de acción motora no parecen estar preparados como tampoco están determinados principalmente por la experiencia, son el resultado de un proceso con dos focos, uno en el sistema nervioso central y otro en las interacciones dinámicas del sujeto con el entorno. El cerebro sin duda tiene una dinámica propia que hace que las neuronas proliferen, migren y se diferencien de determinadas formas y en determinados momentos. Sin embargo, las capacidades de acción emergentes también están moldeadas de manera crucial por las interacciones del sujeto con el medio ambiente (Rosero V, 2016, p. 41). 
Son una función de ambas cosas y surgen de la interacción dinámica entre el cerebro, el cuerpo y el mundo exterior. Otro factor importante del desarrollo es la biomecánica del cuerpo: la percepción, la cognición y la motivación están incorporadas y sujetas a limitaciones biomecánicas. Esas limitaciones cambian drásticamente con la edad y son afectadas por el desarrollo del cerebro y por la forma en que se realizan las acciones (Ayala, et al, 2015).

\section{Proceso de adquisición}

El sistema nervioso se desarrolla de la manera más acelerada durante los primeros meses de vida posnatal (Rosero V, 2016, p. 39). Una vez que se establece una masa crítica de conexiones, comienza un proceso de autoorganización que da como resultado nuevas formas de percepción, acción y cognición, la aparición de nuevas formas de acción siempre se basa en múltiples desarrollos(Clavo M, 2019). Con el desarrollo, los diferentes sistemas de acción también se vuelven cada vez más orientados al futuro y se integran entre sí. En última instancia, cada acción involucra múltiples sistemas de movimientos coordinados que se relacionan con la necesidad de integrarse favorablemente en el entorno.

\section{La motivación como factor fundamental del desarrollo motor}

Los motivos generados internamente son cruciales para la formación de nuevos comportamientos. Por la misma razón, los niños abandonan los patrones de conducta establecidos en favor de otros nuevos (Aceituno F \& Pina H, 2018, p. 26). Los motivos directos son, por supuesto, diferentes. Los niños encuentran placer al explorar sus posibilidades de acción, cuando se abren nuevas posibilidades como resultado, por ejemplo, del establecimiento de nuevas vías neuronales, una mejor percepción o cambios biomecánicos, los niños están ansiosos por explorarlos. Al mismo tiempo, están ansiosos por explorar lo que los objetos y eventos de su entorno ofrecen en términos de sus nuevos modos de acción (Ochoa C, 2019).

\section{Contextos educativos urbano y rural}

Hoy en día, la educación es el aspecto más importante en el desarrollo de la sociedad. La escuela tiene que desempeñar un papel importante para garantizar el éxito de este propósito. En cuanto a los estudiantes, deben desarrollar adecuadamente habilidades y ser proactivos con el propósito de proyectarse productivamente hacia el futuro. Además, los estudiantes deben relacionarse a los aspectos curriculares de forma activa, para alcanzar el mejor rendimiento académico posible (Castillo M, 2018, p. 61).

En este contexto, los entornos y los espacios en los que crecen los estudiantes también desempeñan un papel importante en el aprendizaje influyendo en el desempeño de los estudiantes. Las razones de las variaciones en el rendimiento son la ubicación geográfica, los recursos, la disponibilidad de tecnología y también la calidad de enseñanza de los maestros. 
Recientes investigaciones educativas han examinado las diferencias en el rendimiento comparando la educación rural con la urbana (Salazar C \& Jiménez D, 2020; Hidalgo C \& Villavicencio C, 2017; Maldonado E, 2016; Rodríguez I, 2015). Los antecedentes de los estudiantes también impactan en su desempeño donde se puede ver que muchos de los que viven en áreas rurales tienen bajo rendimiento académico cuando se comparan con los estudiantes que viven en áreas urbanas. Hay muchos factores que causan la brecha de desempeño entre los estudiantes en las áreas rurales y urbanas. (Arichábala C, 2015, p. 13).

De manera general, la información consultada reporta que: el mejor desempeño de los estudiantes urbanos se debe a una mayor calidad en los procesos educativos, conveniente disponibilidad de información que obtienen de diversas fuentes como los medios de comunicación y medios electrónicos, el nivel educativo de las familias de las que proceden suele ser más alto, así como el de las familias de sus pares por lo que se promueve un mejor desempeño, tienen muchas ventajas en infraestructura, más y mejores recursos didácticos (Avendaño $\mathrm{S}, 2018$ ).

La situación actual de la educación inicial en los sectores rurales, es escasamente estudiada, reflejando que no existe el interés de conocer objetivamente la verdadera situación de la forma en que opera, ni como se dan las relaciones entre los diversos factores que influyen en su eficiencia y eficacia (Salazar C \& Jiménez D, 2020, p. 14).

Si bien es cierto, la educación es un instrumento eficaz que desarrolla la confianza, la previsión, la autoestima y la autoeficacia, a los estudiantes que viven en sectores rurales con bajos niveles de desarrollo les resulta complejo obtener la mejor educación para ellos y su entorno de vida se convierte en uno de los factores que obstaculizan su éxito (Clavo M, 2019, p. 8). Se puede asegurar que existe una brecha de desempeño entre los estudiantes que viven en áreas rurales y urbanas debido a diversos factores que contribuyen a las diferencias de desempeño entre los estudiantes de entre los más sobresalientes se puede hacer referencia a los siguientes:

Factor familiar. - En las zonas rurales, la familia es uno de los factores que determina el desempeño de sus hijos. De acuerdo a la información analizada, los estudiantes de áreas rurales tienen bajo rendimiento en comparación con los estudiantes de áreas urbanas porque se relaciona con la educación de sus padres. El entorno familiar es uno de los factores más importantes y de gran influencia para determinar el rendimiento académico de los estudiantes y su vinculación con el desarrollo motor en los primeros años de vida (Reyes A, et al, 2015).

De acuerdo a los resultados propuestos por Maldonado E (2016, p. 3), que los padres rurales no sienten presión para lograr un buen desempeño y las expectativas hacia la educación de sus hijos son bajas. El estímulo de los padres tiene u1na influencia positiva en el desempeño de sus hijos y en las áreas rurales a la mayoría de los padres no les importaba el desempeño académico de sus hijos. Algunos expertos creen que las 
expectativas de los padres son el factor que más influye en las decisiones de los jóvenes de seguir una educación (Zuta T \& Comeca P, 2019).

Factor socioeconómico. - La mayoría de los estudiantes que viven en áreas rurales provienen de familias de bajos ingresos. Sus padres no pueden proporcionarles suficientes recursos educativos en casa (Poblete, Toro, \& Cruzat, 2016). En comparación con los estudiantes de las zonas urbanas, la mayoría de ellos provienen de familias con mejores ingresos por lo que pueden acceder a instituciones educativas que, por sus características permiten obtener un mejor rendimiento. Los estudiantes urbanos tienen la posibilidad de acceder a medios educativos adicionales, mientras que los estudiantes en entornos rurales, no tiene acceso a los mismos recursos educativos afectando su desempeño en comparación con aquellos que tienen acceso a dichos recursos.

\section{Infraestructura y recursos didácticos}

La educación promueve el capital humano como un activo que tiene mayores o menores tasas de retorno dependiendo de la calidad de la educación que se brinde. Algunos de los factores que contribuyen al bajo rendimiento son la falta de recursos y las malas instalaciones en la mayoría de las escuelas, especialmente en las zonas rurales. El problema de la distribución desigual de recursos entre provincias, áreas rurales y urbanas sigue intacto (Alvarez Á \& Ugarte H, 2019). Varias escuelas en áreas rurales no cuentan con los espacios lúdicos y los recursos didácticos adecuados y esta situación significa que los estudiantes aprenden y se desarrollen de forma inadecuada. (Silva Martínez, 2018).

\section{Capacidades docentes.}

El Docente tiene el papel protagónico para lograr un desarrollo adecuado y un buen desempeño en los estudiantes, sin embargo de acuerdo a los datos analizados, la mayoría de los profesores prefieren trabajar en áreas urbanas, antes que en entornos rurales, esto por diversos factores, entre ellos, la posibilidad que la docencia urbana da para el crecimiento en habilidades y aprendizajes metodológicos, mejor remuneración, cercanía a los recursos tecnológicos, la distancia geográfica entre otros (Reyes A, Vaca C, \& Panchi, 2015).

Se ha establecido, que existe una relación directa entre el grado de experiencia docente y la calidad del proceso educativo (Martínez Ch, Guevara A, \& Valler O, 2016). La mayoría de docente en el sector rural aún no domina la técnica de enseñanza porque todavía son nuevos en el área de enseñanza. Por lo tanto, la educación rural cuenta con docentes con las mejores intenciones de trabajo, pero con bajos perfiles de enseñanza, con escasa orientación y medios y recursos educativos escasos de ahí que el progreso de aprendizaje de los estudiantes se vea afectado y no funcione correctamente.

Vinculado este fenómeno con el desarrollo de la motricidad Astoyauri y Danae (2019, p. 89), logran establecer las ventajas de la educación para el desarrollo motriz en sectores rurales, al considerar los altos niveles de movilidad, por la accidentalidad geográfica, las formas de relaciones culturales y sociales y las motivaciones familiares, factores con los 
que cuentan los estudiantes, sin embargo reportan un bajo desarrollo de la motricidad fina, que está vinculada con el desarrollo de habilidades básicas cognitivas, importantes para el desarrollo de la lectura, escritura, lógica y matemática.

\section{Formas de abordar las diferencias de la brecha de logros entre estudiantes rurales y urbanos}

Para superar las diferencias entre los estudiantes de las zonas rurales y urbanas, la literatura consultada propone tomar varias vías entorno a los actores del quehacer educativo, a continuación, se proponen los elementos clave para promover estos procesos y reducir la brecha entre la educación rural y urbana.

Administración educativa - La administración educativa central desempeña un papel importante en la mejora de los resultados educativos de los estudiantes rurales, Debe proporcionar suficientes instalaciones a centros educativos ubicadas en áreas rurales iguales o similares a las de los centros educativos urbanos. Además de eso, el gobierno también debe brindar un apoyo financiero adicional a los centros educativos que están en las áreas rurales donde se sabe que la mayoría de los estudiantes provienen de familias de bajos ingresos y no poseen los medios suficientes como para obtener una mejor educación y servicios.

Es importante que los organismos pertinentes se aseguren de que los docentes estén adecuadamente calificados para enseñar las asignaturas que se les ha asignado y que puedan hacer su trabajo correctamente, esto tiene mayor relevancia en la educación inicial, donde los procesos de desarrollo deben ser tratados con mayore delicadeza y cuidado, para desarrollar las habilidades y nociones básicas necesarias para que los estudiantes tengan un desempeño eficiente en los años posteriores (Garzón M, 2019, p. 9).

Papel de los padres. - Mientras los maestros desempeñan su papel en la escuela, los padres deben desempeñar sus roles en casa. Aunque la mayoría los padres de las zonas rurales no tienen una educación adecuada (Calderón C, 2015), pueden ayudar a sus hijos a desempeñarse en términos de brindarles apoyo moral y motivación para aprender. Sin embargo, de que ayudar en términos de proporcionar recursos para promover los aprendizajes, los padres deben darse cuenta de lo importante que tiene la educación para sus hijos y su futuro. Es importante además que los padres estén constantemente informados sobre los progresos de sus hijos para realizar un adecuado acompañamiento durante el proceso educativo, preocupándose siempre por el desempeño monitoreando su progreso.

El papel de los Docentes. - El docente, es una fuerza importante en el aprendizaje, Su trabajo es la fuente que ayuda a superar el problema del bajo rendimiento entre los estudiantes rurales. Su misión es la de promover y desarrollar aprendizajes durante el tiempo de estudio, alentando a los estudiantes a participar en las actividades programadas y desarrollar su autoestima. Además, el docente debe crear un ambiente de apoyo y 
cuidado para que los estudiantes se sientan cómodos durante su aprendizaje (Avendaño S, 2018).

Las Instituciones Educativas en contextos rurales y urbanos se caracterizan por sus fortalezas y debilidades específicas. De acuerdo a Clavo (2019, p. 18), Las escuelas rurales y urbanas son muy parecidas cuando se trata planes y programas de estudio. Sin embargo, existen diferencias significativas que afectan el rendimiento de los estudiantes y que están relacionadas directamente a los factores del entorno. Sin embargo, esta situación no es insalvable, es posible que las soluciones no sean fáciles, pero se requiere un alto grado de compromiso y una comprensión racional del entorno.

En la literatura consultada (Salazar C \& Jiménez D, 2020; Clavo M, 2019; Zuta T \& Comeca P, 2019; Rosero V, 2016; Rodríguez I, 2015), sugieren que para mejorar el desempeño de los estudiantes rurales se deben comprender adecuadamente su entorno y las prioridades sociales, culturales y económicas de los habitantes, mantener los valores internos como la autoeficacia y la autoestima, De este análisis se puede concluir que existen diferencias entre el desempeño de los estudiantes en las escuelas rurales y urbanas. Los estudiantes que viven en áreas urbanas obtendrán oportunidades de excelencia de alto rendimiento que les brinda su ubicación. Los estudiantes urbanos tienen mayor acceso a muchos recursos y, por lo tanto, tienen oportunidades que no son fácilmente accesibles para los estudiantes rurales. Por otro lado, generalmente, los padres urbanos tienen un mayor nivel de escolaridad por lo que están más conscientes de las ventajas de la educación. Los padres de estudiantes rurales tenían menos expectativas.

\section{Metodologia.}

La investigación propuesta tiene un enfoque cuanti-cualitativo, comparándose las realidades del desarrollo motor en los dos contextos mencionados y explicándose después las diferencias entre ellos, el diseño de la investigación es secuencial, de tipo descriptiva, responde a la hipótesis, "El desarrollo de la motricidad es significativamente diferente en niños de 4 a 5 años en contextos urbanos con respecto a niños en contextos rurales". La investigación para establecer comparativamente el desarrollo motriz de niños menores de 5 años en sectores rurales y urbanos, implicó la fundamentación científica de las variables en estudio, para el efecto se realizó una investigación bibliográfica de textos vinculados al tema a utilizando buscadores de información académica en internet, se seleccionaron 40 documentos entre informes de investigación y artículos científicos de los cuales se escogieron 12 en los cuales se encontró vinculación directa con las variables en estudio.

La identificación de los niveles de motricidad alcanzados por los niños de 5 años de los sectores urbanos y rurales, se trabajó en 160 niños de entre 4 y 5 años pertenecientes a tres centros educativos urbanos y tres rurales, los centros educativos fueron seleccionados al azar, se realizó un a inducción con los padres de familia, los cuales firmaran un consentimiento informado, se llenaron fichas sociodemográficas de cada estudiante y se les aplico el Test Escala Abreviada del Desarrollo de Nelson Ortiz (2005) que por sus características permitió evaluar los niveles de motricidad fina y gruesa y la posible 
existencia de algún tipo de retraso en el desarrollo motriz, para el efecto, se realizaron las actividades que establece el test, asignándole a cada una de ellas un puntaje, la suma del puntaje ubica al estudiante en uno de cuatro niveles, Alerta, Medio, Medio Alto y Alto, determinado de esta manera el nivel de desarrollo de motricidad que le caracteriza, los datos obtenidos se sistematizaron en tablas y gráficos para su análisis.

Se buscó comprobar el supuesto hipotético de que existen diferencias significativas en los niveles de motricidad gruesa y fina de los estudiantes de 4 y 5 años pertenecientes a los sectores urbanos y rurales. Para el efecto, se realizó una prueba estadística de t de Student para muestras independientes, utilizando los datos obtenidos del Test aplicado, considerando que se comparan los niveles de motricidad de los niños de los sectores urbano y rural, la prueba se aplicó para la variable de motricidad gruesa y para variable de motricidad fina. Para el análisis de los factores que influyen en el desarrollo de la motricidad en los estudiantes en estudio, se aplicó un cuestionario a los 6 docentes de los estudiantes y se trabajó con la metodología de Grupo Focal con los actores clave del proceso educativo vinculado al nivel educativo en cuestión, los resultados obtenidos de la aplicación de estos instrumentos se sistematizaron utilizando un registro anecdótico y correlacionando los criterios de los docentes finalmente los hallazgos encontrados fueron socializados con los docentes de los centros educativos en donde se realizó la intervención y se socializaron a nivel general.

\section{Resultados.}

La caracterización de la muestra utilizada para el análisis de la motricidad de los niños de educación inicial de los sectores urbanos y rurales es la siguiente:

\begin{tabular}{lllllll}
\hline & \multicolumn{5}{l}{ Sexo } & \multicolumn{3}{c}{ Total } \\
\cline { 2 - 5 } Entorno & Hombres & \multicolumn{4}{c}{ Mujeres } \\
\cline { 2 - 6 } & $\mathrm{f}$ & $\%$ & $\mathrm{f}$ & $\%$ & $\mathrm{f}$ & $\%$ \\
\hline Urbano & 43 & $53,8 \%$ & 37 & $46,3 \%$ & 80 & $100,0 \%$ \\
Rural & 53 & $66,3 \%$ & 27 & $33,8 \%$ & 80 & $100,0 \%$ \\
\hline Total & 96 & $60,0 \%$ & 64 & $40,0 \%$ & 160 & $100,0 \%$ \\
\hline
\end{tabular}

Tabla 1 Distribución por sexo en niños de 48 a 71 semanas en Instituciones Educativas Urbanas y Rurales.

Fuente: Registros Escolares

De los 160 niños en edades comprendidas entre 48 a 71 semanas que asisten a los niveles de educación inicial, el $60 \%$ son hombres y el $40 \%$ mujeres, para el sector Urbano el $53,8 \%$ de los niños son hombres y el 46,3\% mujeres mientras que en el sector rural $66,3 \%$ hombres y 33,8\% mujeres, este resultado indica que la población de niños que asisten a las instituciones educativas a la educación inicial son más varones que mujeres, sobre 
todo en el sector rural en donde las diferencias porcentuales son más acentuadas como se puede observar en la tabla 1

\begin{tabular}{|c|c|c|c|c|c|c|c|c|c|c|}
\hline \multirow{3}{*}{ Entorno } & \multicolumn{8}{|c|}{ Edades en meses } & \multirow{2}{*}{\multicolumn{2}{|c|}{ Total }} \\
\hline & \multicolumn{2}{|c|}{$\begin{array}{l}<=48 \\
\text { meses }\end{array}$} & \multicolumn{2}{|c|}{ 49-59 meses } & $\begin{array}{l}60 \quad a \\
\text { meses }\end{array}$ & 70 & \multicolumn{2}{|c|}{$\begin{array}{l}71= \\
\text { meses }\end{array}$} & & \\
\hline & $f$ & $\%$ & $f$ & $\%$ & $f$ & $\%$ & $f$ & $\%$ & $f$ & $\%$ \\
\hline Urbano & 3 & $3,8 \%$ & 46 & $57,5 \%$ & 30 & $37,5 \%$ & 1 & $1,3 \%$ & 80 & $100,0 \%$ \\
\hline Rural & 5 & $6,3 \%$ & 41 & $51,2 \%$ & 33 & $41,3 \%$ & 1 & $1,3 \%$ & 80 & $100,0 \%$ \\
\hline Total & 8 & $5,0 \%$ & 87 & $54,4 \%$ & 63 & $39,4 \%$ & 2 & $2,6 \%$ & 160 & $100,0 \%$ \\
\hline
\end{tabular}

Tabla 2 Distribución por edad en meses de los niños en los sectores urbanos y rurales Fuente: Registros Escolares

La distribución de los niños por edades de las instituciones educativas urbanas y rurales es la siguiente: $54,4 \%$ son niños de entre 49 y 59 meses, el 39,4\% se ubican entre los 60 y 70 meses solo el 5\% es igual a 48 meses y apenas 1,3\% es igual o mayor a 71 meses. La distribución para cada sector indica que las edades de los niños en el sector urbano son del $57,5 \%$ de 49 a 59 meses $37,5 \%$ de 60 a 70 meses, 3,8\% iguales a 48 meses y 1,3 iguales a 71 meses. En el sector rural la distribución por edad es del 51,2\% en la edad de 49 a 59 meses, el $41,3 \%$ entre 60 y 70 meses igual a 48 meses el 6,3\% e igual a 71 meses $1,3 \%$, lo que indica una mayor presencia de estudiantes en la edad de 49 meses a 59 meses, seguramente por la incidencia de matrícula, estos resultados se pueden observar en la tabla 2.

Los resultados obtenidos de la aplicación del Test Escala Abreviada del Desarrollo de Nelson Ortiz para motricidad gruesa de acuerdo a los entornos urbano y rural se detallan en la tabla 3.

\begin{tabular}{|c|c|c|c|c|c|c|c|c|c|c|}
\hline \multirow{3}{*}{ Entorno } & \multicolumn{8}{|c|}{ Puntajes } & \multirow{2}{*}{\multicolumn{2}{|c|}{ Total }} \\
\hline & \multirow[t]{2}{*}{ 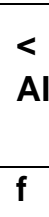 } & ta $=26$ & \multicolumn{2}{|c|}{$\begin{array}{l}\text { 27-29 } \\
\text { Medio }\end{array}$} & $\begin{array}{l}30-32 \\
\text { Alto }\end{array}$ & Medio & \multicolumn{2}{|c|}{$33=>$ Alto } & & \\
\hline & & $\%$ & $f$ & $\%$ & $f$ & $\%$ & $f$ & $\%$ & $f$ & $\%$ \\
\hline Urbano & 6 & $7,5 \%$ & 50 & $62,5 \%$ & 20 & $25,0 \%$ & 4 & $5,0 \%$ & 80 & $100,0 \%$ \\
\hline Rural & 4 & $5,0 \%$ & 22 & $27,5 \%$ & 49 & $61,3 \%$ & 5 & $6,3 \%$ & 80 & $100,0 \%$ \\
\hline Total & 10 & $6,3 \%$ & 72 & $45,0 \%$ & 69 & $43,1 \%$ & 9 & $5,6 \%$ & 160 & $100,0 \%$ \\
\hline
\end{tabular}

Tabla 3 Puntajes de Test de Escala Abreviada del Desarrollo para motricidad gruesa

Fuente: Resultados de Test de Escala Abreviada del Desarrollo para motricidad gruesa 
Del total de estudiantes analizados el $45 \%$ alcanza un desarrollo motriz grueso medio, el $43,1 \%$ un desarrollo motriz medio alto, el 6,3\% se encuentra en estado de alerta, mientras que el 5,6\% del total de niños tiene un alto nivel de desarrollo motriz grueso. De forma específica para el sector urbano el nivel de desarrollo de la motricidad gruesa alcanza su mayor porcentaje $62,5 \%$ en el nivel medio, logran un $25 \%$ para el nivel medio alto, se reporta el 7,5\% en el nivel de alerta y el $5 \%$ en el Nivel Alto. Para el sector rural el desarrollo motor grueso alcanza su mayor porcentaje en el nivel medio alto con $61,3 \%$ de los niños, el 27,5\% corresponde al nivel medio, 6,3\% al nivel alto y el 5\% en Alerta, como se puede observar en la tabla 4

La evaluación estadística de estos resultados indica que los estudiantes del sector rural alcanzan un mayor nivel de desarrollo motriz grueso que los estudiantes del sector urbano, estableciéndose una diferencia importante entre los estudiantes que alcanza un desarrollo motriz medio alto, en donde los niños rurales demuestran un mayor desarrollo.

\begin{tabular}{|c|c|c|c|c|c|c|c|c|c|c|}
\hline \multirow{3}{*}{ Entorno } & \multicolumn{8}{|c|}{ Puntajes } & \multirow{2}{*}{\multicolumn{2}{|c|}{ Total }} \\
\hline & \multicolumn{2}{|c|}{$\begin{array}{l}<=23 \\
\text { Alerta }\end{array}$} & \multicolumn{2}{|c|}{$\begin{array}{l}\text { 24-28 } \\
\text { Medio }\end{array}$} & \multicolumn{2}{|c|}{$\begin{array}{l}\text { 29-33Medio } \\
\text { Alto }\end{array}$} & \multicolumn{2}{|c|}{$34=>$ Alto } & & \\
\hline & $f$ & $\%$ & $f$ & $\%$ & $f$ & $\%$ & $f$ & $\%$ & $f$ & $\%$ \\
\hline Urbano & 14 & $17,5 \%$ & 19 & $23,8 \%$ & 39 & $48,8 \%$ & 8 & $10,0 \%$ & 80 & $100,0 \%$ \\
\hline Rural & 16 & $20,0 \%$ & 29 & $36,3 \%$ & 29 & $36,3 \%$ & 6 & $7,5 \%$ & 80 & $100,0 \%$ \\
\hline Total & 30 & $18,8 \%$ & 48 & $30,0 \%$ & 68 & $42,5 \%$ & 14 & $8,8 \%$ & 160 & $100,0 \%$ \\
\hline
\end{tabular}

Tabla 4 Puntajes de Test de Escala Abreviada del Desarrollo para motricidad fina Fuente: Resultados de Test de Escala Abreviada del Desarrollo para motricidad gruesa

Los resultados obtenidos de la aplicación del test de Escala abreviada del desarrollo para la motricidad fina se proponen en la tabla 4, encontrándose que de los 160 estudiantes analizados el $42,5 \%$ alcanza un desarrollo motriz fino medio alto, el $30 \%$ se ubica en el nivel de medio el $18,8 \%$ de los niños se encuentra en un nivel de alerta y el 8,8 en un nivel alto. De forma particular en el entorno urbano se reporta que el $48,8 \%$ de los estudiantes analizados alcanza un nivel de desarrollo motor fino medio alto, el 23,8\% alto $17,5 \%$ se encuentran en un nivel de alerta y el $10 \%$ en un nivel Alto. En el entorno Rural se reporta que para los niveles medio alto y alto los estudiantes alcanzan el 36,3\% para cada nivel, el $20 \%$ se encuentran en un nivel de alerta y el 7,5 alcanza un nivel alto.

De este resultado se infiere que los estudiantes del sector urbano tienen un mejor desarrollo de la motricidad fina, pero las diferencias no son tan contundentes en relación con la evaluación de la motricidad gruesa, otro aspecto a considerar en esta evaluación es el importante porcentaje de estudiantes evaluados tanto en el sector urbano como en el 
sector rural que se encuentran en el nivel de alerta y medio, demostrando que se requiere realizar un mayor esfuerzo para desarrollar estas habilidades en los dos entornos.

A continuación, se responde a la hipótesis planteada en la investigación que supone que existen diferencias significativas en el desarrollo motriz de los estudiantes de 4 y 5 años de los entornos urbano y rural para el efecto se sometieron los datos obtenidos a la prueba estadística de $\mathrm{T}$ de student para muestras independientes, donde los resultados obtenidos fueron los siguientes:

\begin{tabular}{llllll}
\hline & & & & Desv. & $\begin{array}{l}\text { Desv. Error } \\
\text { promedio }\end{array}$ \\
\hline Puntaje & Motricidad & Urbano & $\mathrm{N}$ & Media & Desviación \\
Gruesa & Rural & 80 & 28,75 & 2,270 &, 254 \\
\hline
\end{tabular}

Tabla 5 Estadísticas del grupo, puntaje de motricidad gruesa

Fuente: Registros Escolares

\begin{tabular}{|c|c|c|c|c|c|}
\hline & & Sig. & Diferencia de & Diferencia de & $\begin{array}{l}95 \% \text { de intervalo de } \\
\text { confianza de } \\
\text { diferencia }\end{array}$ \\
\hline $\mathbf{t}$ & gl & (bilateral) & medias & error estándar & Inferior Superior \\
\hline$-3,906$ & 158 & ,000 & $-1,363$ & ,349 & $-2,051$ \\
\hline
\end{tabular}

Tabla 6 Resultados de t student para muestras independientes motricidad gruesa.

Fuente: Registros Escolares

De acuerdo a los resultados obtenidos se encuentra que existen diferencias significativas en el desarrollo de la motricidad gruesa en el entorno urbano con una media de 28,75 y un error estándar de 0,254 con respecto al entorno rural con una media de 30,11 y un error estándar de 0,239 y un valor de t 3,916 con 158 grados de libertad con un valor de P 0,000 $<0,05$ y una potencia (r) de 0,75 (grande)

\begin{tabular}{llllll}
\hline & & & & Desv. & $\begin{array}{l}\text { Desv. Error } \\
\text { promedio }\end{array}$ \\
\hline Puntaje & Motricidad Untorno & N & Media & Desviación & 80 \\
Fina & Rural & 80 & 28,83 & 4,151 &, 464 \\
\hline
\end{tabular}

Tabla 7 Estadísticas de grupo, puntaje de motricidad fina

Fuente: Registros Escolares 
Considerando los resultados obtenidos se encuentra que no existen diferencias significativas en el desarrollo de la motricidad fina en el entorno urbano con una media de 28,83 y un error estándar de 0,464 con respecto al entorno rural con una media de 27,91 y un error estándar de 0,429 y un valor de t 1,445 con 158 grados de libertad con un valor de $\mathrm{P} 0,150>0,05$ y una potencia (r) de 0,114 (grande).

\section{Factores socio educativos que limitan el desarrollo de la motricidad en los entornos urbano y rural}

Los Factores socio educativos en el que se desenvuelven los docentes de los niños de educación inicial, se obtuvieron de la aplicación de una encuesta validada por tres expertos en desarrollo motriz y dio lugar a la determinación de los siguientes factores de influencia:

\section{Sobre los contextos educativos en el que labora}

Al hablar del contexto educativo en donde laboran los docentes de los centros educativos urbanos confirman que sus instituciones son cerradas y que no cuentan con los espacios necesarios como para realizar las actividades de aprendizaje como ellos quisieran, las condiciones de las instalaciones no son las más propicias y resulta complejo trasladarse a otros sitios debido al alto grado de responsabilidad que esto implica.

Los docentes de los sectores rurales, aseguran que a pesar de que sus escuelas están en poblaciones de pocos habitantes y gozan de un ambiente natural próximo, están sujetos a limitantes, que al igual que en las instituciones urbanas están ligados al grado de responsabilidad que implica para los docentes desarrollar actividades extra clase con los niños, lo que reduce las capacidades de los docentes para aprovechar estos entornos.

\section{Ventajas y desventajas del contexto en donde usted labora en el desarrollo motor de los niños.}

Para los docentes del sector urbano las ventajas están dadas por los servicios a los que pueden acceder de forma inmediata, las instituciones educativas además de los servicios básicos cuentan con otros complementarios como internet, transporte público y las instituciones de apoyo, además que en la ciudad las necesidades de recursos educativos estructurados pueden suplirse con mayor facilidad, puesto los productos y servicios se ofertan en el mercado local y son de fácil acceso. En cuanto a las desventajas los docentes del entorno urbano consideran a los espacios de trabajo, la inseguridad ciudadana, la contaminación ambiental, el encierro al que son sometidos los estudiantes luego de salir de clases y el individualismo que genera un alto grado la falta de colaboración de los padres de familia.

En el sector rural los docentes, consideran que una de las ventajas más importantes es el espacio con el que cuentan, la contaminación por ruido, y emisión de gases tóxicos es prácticamente nula, se cuenta con los servicios básicos, agua, luz, el internet es limitado y la señal baja. Para los docentes rurales los aspectos negativos se centran en la dificultad 
para cubrir necesidades específicas de la institución debido a las distancias que tienen que recorrer los docentes y los estudiantes, existe participación de los padres a las reuniones, pero escasa colaboración, se tiene que trabajar con los escasos recursos que se posee.

\section{El currículo es el adecuado al momento de impartir sus clases}

En relación al currículo con el que se trabaja los docentes urbanos consideran que en algunos aspectos es adecuado, pero que en otros existen contradicciones, sobre todo en las actividades sugeridas, para las cuales se requiere un acercamiento a espacios abiertos y recursos educativos específicos que la institución no posee, por otro lado se plantean actividades que están fuera de los contextos socio económicos en los que se desarrollan los niños, teniendo los docentes que ajustarse de cualquier manera para cumplir estos requerimientos.

Para los profesores del sector rural la malla curricular está diseñada más para los sectores urbanos, por lo que resulta complejo cumplir con las actividades, por otro lado, la realidad socioeconómica de las poblaciones no se vincula casi en nada a las mallas curriculares que parecen haber sido diseñadas por personas que jamás tuvieron una a experiencia docente a nivel rural.

\section{Correspondencia entre contenidos curriculares para la enseñanza de la motricidad y requerimiento de loas actores}

Los docentes urbanos consideran que los contenidos propuestos en la malla curricular son más teóricos que prácticos por lo que se hace difícil que los niños desarrollen buenos niveles de motricidad y competencias de aprendizaje. Para los docentes rurales los contenidos y las actividades no se corresponden a las necesidades de los niños y sus entornos, no han sido pensados en las realidades locales por lo que, los docentes aseguran que tienen que adaptarlos

\section{Incidencia del entorno físico en el desarrollo de la motricidad}

De acuerdo a la percepción de los docentes del sector urbano los elementos físicos que se pueden encontrar en el ambiente de aprendizaje si influyen en el desarrollo motriz, sin embargo, establecen una diferencia importante en entre aquellos elementos que son necesarios para el desarrollo de la motricidad fina y aquellos que son fundamentales para la motricidad gruesa aseguran que los elementos de infraestructura para el desarrollo de la motricidad gruesa son escasos y limitados en el sector urbano.

Por su parte los docentes rurales coinciden con los criterios de los docentes urbanos en cuanto a la importancia de los espacios físicos, y las diferencias entre estos recursos para desarrollar las motricidades finas o gruesos, según sea el caso, sin embargo aseguran que a pesar de encontrase próximos a los espacios abiertos, se requieren de elementos que llamen la atención de los niños para que estos desarrollen habilidades motrices gruesas específicas, aseguran que son escaso los elementos estructurados de que disponen, lo mismo aseguran para el desarrollo de la motricidad fina, explicando que no poseen los 
recursos didácticos suficientes para cumplir con los objetivos propuestos en las mallas curriculares.

\section{Nivel de desarrollo motriz de los estudiantes en las instituciones}

Con respecto a la pregunta sobre el actual nivel de desarrollo de la motricidad en los estudiantes de educación inicial de las instituciones evaluadas en los entornos urbanos y rurales, la percepción de los docentes es la siguiente.

En los dos entornos los docentes coinciden que las evaluaciones son superficiales y se hacen en función de las capacidades adquiridas que se miden en función de los lineamientos de la malla curricular, sin embargo este tipo de evaluación no es suficiente como para establecer un nivel adecuado de motricidad, de tal suerte que la evaluación de la motricidad gruesa generalmente se le hace en función de actividades de educación físicas que el estudiante realiza, sobre todo en el cumplimento de las horas de clase de educación física, de igual manera se pretende desarrollar habilidades motoras finas, en los niños como pre requisito para cubrir las necesidades posteriores de escritura y lectura, pero los docentes no conocen las formas adecuadas de determinar los niveles alcanzados por los niños y el avance de los procesos se da en función de lo propuesto en la planificación curricular obligatoria.

\section{Calidad de los recursos educativos de su entorno para la enseñanza}

En el caso de los dos entornos, evaluados, los docentes coinciden en que carecen de los recursos necesarios para un buen desarrollo de la motricidad en sus estudiantes, esto es en el proceso de elaboración, ejecución y evaluación de las actividades, aseguran que es muy esporádica la incorporación de recursos didácticos apropiados y nuevos. En este sentido en los dos entornos, los centros educativos o carecen de material o el material que poseen está obsoleto. En los dos entornos los docentes aseguran que para poder cumplir con su trabajo en aula ellos mismos deben elaborar los materiales y recursos de su gasto, por lo que no hacen un gran esfuerzo en renovar estos materiales.

Por otro lado, aseguran que las veces en las que se les ha entregado recursos y materiales, en su mayoría no cumplían con los requerimientos necesarios como para constituirse en adecuadas herramientas de enseñanza, debido a que, o no estaban elaborados con buenos materiales, no correspondían a los procesos curriculares para el nivel diseñados por los organismos oficiales, o se les daba el material sin ningún tipo de capacitación o inducción.

\section{Análisis Grupo Focal}

El grupo focal estuvo integrado por los docentes de educación inicial que se encuentra a cargo de los niños analizados en el estudio, tres de entorno urbano y tres de entorno rural, dos expertos en didáctica y pedagogía que participaron en la validación de la encuesta. Se trataron aspectos importantes vinculados a las diferencias del desarrollo motor en estudiantes de educación inicial. Los resultados alcanzados en este ejercicio colaborativo los siguientes: 
Se estableció que tanto para los docentes de los sectores urbanos como para los docentes rurales evaluar los niveles de motricidad resulta complejo, debido a que desconocen de un modelo adecuado para hacerlo

Establecieron que ellos tratan de cumplir con los requerimientos exigido por la formalidad de la malla curricular, cuya estructuración viene ya determinada por los lineamientos impuestos por los organismos gubernamentales competentes.

Los docentes urbanos aseguran no poseer los espacios suficientes para promover el desarrollo de la motricidad gruesa y también son escasos los recursos para promover el desarrollo de la motricidad fina. Los docentes rurales establecen que si bien es cierto los espacios abiertos son cercanos a los establecimientos educativos, realizar actividades que estén fuera de la institución implica un alto grado de responsabilidad y son los mismos representantes educativos los que las prohíben, por otro lado, aseguran no tener al interior de las instituciones los medios necesarios para fomentar el desarrollo motriz grueso ni los recursos para hacer lo propio con el desarrollo motriz fino.

Los docentes urbanos concuerdan en el hecho de que las actividades que realizan se orientan de mayor manera a las actividades de motricidad fina preparatorias para facilitar los procesos posteriores de enseñanza de la escritura y la lectura, el desarrollo motor grueso se pretende realizar a través de actividades lúdicas y en las clases de educación física, sin embargo aceptan que con el pasar del tiempo los niños urbanos tiene cada vez más problemas en el desarrollo de la motricidad gruesa, que también incide en el desarrollo de la motricidad fina, sin embargo el desarrollo de la primera es mucho menor que el de la segunda.

Los docentes rurales concuerdan en el hecho de las dificultades que deben enfrentar para tratar de consolidar los niveles de motricidad exigidos en la planificación, aseguran que si los niños tienen una excelente motricidad gruesa es debido a su forma de vida, ya que constantemente están realizando actividades que en las que se ponen de manifiesto habilidades motoras finas (correr, saltar, etc.), sin embargo les resulta complejo realizar actividades motrices gruesas vinculadas con la integración sensorial como bailar o marchar.

En el conversatorio las docentes y los especialistas participantes, concluyen que es bastante complejo establecer los niveles de motricidad de los niños y que resulta complejo establecer parámetros referenciales para comparar el desarrollo motor entre los niños de los entornos urbanos con respecto a sus pares de los entornos rurales.

Sin embargo coinciden en que definitivamente los entornos están influyendo en el desarrollo motor ya que los espacios en los sectores urbanos implican mayor trabajo e $n$ los aspectos motores finos orientados a fortalecer los procesos posteriores de lectura y escritura, y las limitaciones de las infraestructura física que limita las actividades motrices gruesas, mientras que los estudiantes de los entornos rurales han desarrollado la motricidad gruesa por el hecho de que tienen que realizar actividades vinculadas a la utilización de movimientos gruesos, sin embargo resulta complejo consolidar la 
motricidad fina debido a que los intereses de los niños están orbitados más a juegos y trabajos al aire libre, sin que esto sea necesariamente parte de los procesos de enseñanza aprendizaje.

\section{Discusión}

De acuerdo al análisis realizado en esta investigación el desarrollo motor está sujeto a factores externos e internos que influyen directa e indirectamente en su aprendizaje, determinar de forma general cuales son estas diferencias implica buscar los problemas de forma muy particular, mucho más aún si se busca establecer criterios que posibiliten establecer un estándar que establezca diferencias y similitudes en las escuelas urbanas y rurales, situación que se comprueba en el siguiente párrafo en el que se comparan dos investigaciones similares con los resultados obtenidos en este trabajo.

Silva (2018) reporta un $11 \%$ de rezago en el desarrollo de la motricidad gruesa en las escuelas rurales, sin embargo, los estudiantes de las escuelas de los entornos urbanos mantienen un desarrollo normal. Rodríguez (2015), indica en su investigación sobre habilidades básicas, motricidad y lenguaje en niños de 3 años que no existen diferencias importantes en relación al entorno en el que viven los estudiantes, esta diferencia se presenta en un porcentaje de 5,5\%, en esta investigación se indica un déficit de motricidad gruesa $7,5 \%$ en las escuelas urbanas y de $5 \%$ en las escuelas rurales, con respecto a la motricidad fina en cambio el déficit es de $17,5 \%$ para los entornos urbanos y $20 \%$ en los sectores rurales, lo que implica.

No se encontró información reportada que estableciera un análisis de diferencias de medias para establecer si el déficit de desarrollo motor se da en función del entorno en el que viven los estudiantes, sin embargo en este trabajo se estableció que si existen diferencias significativas de desarrollo motriz grueso en estudiantes de entornos urbanos y rurales, sin embargo en contraposición al resultado de Silva (2018), el déficit de desarrollo de motricidad gruesa es menor en el sector rural que en el urbano, por otro lado y concordando con Rodríguez no existe diferencias significativas en lo que respecta a motricidad fina, sin embargo el análisis descriptivo revela una ventaja porcentual del sector urbano sobre el rural en la adquisición y desarrollo de esta motricidad.

\section{Conclusiones.}

- Del desarrollo motor depende la adaptación de los individuos a sus entornos, este proceso se da en función de las necesidades que tienen que ser satisfechas para permitir la supervivencia del individuo, esto se traduce de forma análoga al proceso educativo, en donde el desarrollo de las habilidades motoras gruesas y finas en los primeros años de vida y en el educación inicial, implica potenciar las capacidades de los estudiantes para solventar de forma eficiente los retos que el aprendizaje de la lectura y la escritura requieren en el futuro. 
- Otro aspecto importante del desarrollo motor está vinculado con la capacidad de sociabilidad que el niño debe tener para adaptarse a su entorno y ser aceptado, los movimientos, las habilidades y las destrezas deben considerarse como parte del proceso de adaptabilidad y de hecho son situaciones que los demás observan y aprueban permitiendo que a través de estas competencias adquiridas el niño se adapte de mejor manera al contexto social.

- El desarrollo natural de la motricidad tiene mucho que ver con las necesidades que el individuo debe satisfacer para así en su entorno, por lo que establecer si las habilidades motrices son diferentes en entornos urbanos y rurales es relativo esto va a depender de las circunstancias en las que el individuo deba desenvolverse y un ejemplo que evidencia lo dicho son el resultados en esta investigación, en donde la motricidad gruesa esta mejor desarrollada en estudiantes del sector rural que en estudiantes de los entornos urbanos; mientras que la motricidad fina es más prevalente en los estudiantes del sector urbano en función de las características de sus entornos de aprendizaje en los que se da mayor relevancia al desarrollo de la parte cognitiva por lo que los niños se ven en la necesidad de aprender con mayor rapidez la lectura y la escritura, habilidades más vinculadas a este tipo de motricidad.

- Se ha demostrado, por las evidencias presentadas, que el desarrollo de la motricidad se da en función de los requerimientos de adaptación que los entornos requieren de los individuos, sin embargo los requerimientos de escolarización no consideran estos aspectos y pretenden estandarizar los procesos de enseñanza aprendizaje en los dos ecosistemas y consideran de importancia vital que los estudiantes se desarrollen de forma uniforme para dar cumplimiento a las estructuras curriculares y a los procesos que marcan los contenidos obligatorios. De ahí que los aspectos ecológicos, socio demográficos y didáctico pelágicos se contraponen entre si y no facilitan una adecuada orientación hacia los maestros para obtener de sus estudiantes aprendizajes motrices significativos y orientados a dar solución a sus problemas más importantes de acuerdo a sus propias realidades.

\section{Conflicto de Intereses}

Los contenidos y los resultados propuestos en esta investigación no tienen ningún tipo de conflicto de intereses.

\section{Referencias bibliográficas.}

Aceituno F, R., \& Pina H, F. (2018). Didáctica de la Motricidad en la formación de profesores de educación infantil. Retos: Nuevas Tendencias en eduacación, 34, 25-32. Obtenido de https://dialnet.unirioja.es/servlet/articulo?codigo=6736313 
$\overline{\text { Alvarez Á, M., \& Ugarte H, A. (2019). La escuela graduada en el medio rural. Un estudio }}$ de caso. Revista de Educación de la Universidad de Granada(26), 163-181. Obtenido de https://repositorio.unican.es/xmlui/handle/10902/18076

Arichábala C, G. (2019). La calidad educativa y el nivel de desarrollo evolutivo de los niños y niñas de 3 a 5 años que asisten a los centros de desarrollo infantil de la zona rural del Cantón Gualaceo. Cuenca: Universidad del Azuay. Obtenido de http://dspace.uazuay.edu.ec/handle/datos/4095

Astoyauri , A., \& Danae, V. (2019). Ventajas del medio rural andino en el desarrollo psicomotor en niños y niñas de 3 y 4 años. Lima: Universidad San Ignacio de Loyola. Obtenido de http://200.37.102.150/handle/USIL/9185

Avendaño S, R. (2018). En niños y niñas de 4-5 años de edad, ¿ Cómo varía el desarrollo psicomotor entre los que asisten a escuelas urbanas y rurales? Estudio en cinco escuelas urbanas y cinco escuelas rurales públicas del cantón Cuenca. Cuenca: Universidad del Azuay. Obtenido de http://dspace.uazuay.edu.ec/handle/datos/4940

Ayala E, J., Arboleda , R., \& Souza, S. (2015). Motricidad y enseñanza: saberes que se transmiten en el aula. Revista Latinoamericana de Estudios Educativos, 11(2), 168-189. Obtenido de https://www.redalyc.org/pdf/1341/134146842008.pdf

Calderón C, A. (2015). Situación de La Educación Rural en el Ecuador. Quito : Centro Latinoamericano para el Desarrollo Rural .

Castillo M, C. (2018). Análisis comparativo del proceso de aprendizaje de la lectoescritura en dos contextos educativos diferentes. Morelos: Universidad Autónoma del Estado de Morelos. Obtenido de http://riaa.uaem.mx/xmlui/bitstream/handle/20.500.12055/448/CAMCNN04T.p df? sequence $=1$

Clavo M, T. (2019). Diferencias en el desarrollo psicomotor en niños de tres a cinco años. Zona rural y urbana de Lajas, Chota 2017. Chota: Univercidad Nacional Autonoma del Chota. Obtenido de https://www.researchgate.net/profile/Ciria_Salazar/publication/340362574_Eval uacion_del_tiempo_efectivo_de_la_clase_de_educacion_fisica_en_escolar_del_ ambito_rural/links/5e84e290299bf1307970c89d/Evaluacion-del-tiempoefectivo-de-la-clase-de-educacion-fisi

Garófano V, V., \& Guirado C, L. (2017). Importancia de la motricidad para el desarrollo integral del niño en la etapa de educación infantil. EmásF: Revista Digital de Eduacación Física(47), 89-105. Obtenido de https://dialnet.unirioja.es/servlet/articulo?codigo $=6038088$

Garzón M, I. (2019). Caracterización de las habilidades básicas motrices en los estudiantes de 5 a 11 años de la básica primaria delo contexto rural en el 
municipio de Guateque Boyacá. Boyaca: Universidad Pedagógica Nacional. Obtenido de http://200.119.126.32/handle/20.500.12209/10078

Hidalgo C, M., \& Villavicencio C, V. (2017). Estudio comparativo del desarrollo en niños de 4 a 5 años, en Centros de Educación Inicial públicos del sector rural y Centros de Educación Inicial privados del sector urbano. Cuenca: UNiversidad del Azuay. Obtenido de http://201.159.222.99/handle/datos/7075

Maldonado E, J. (2016). Eficacia de la estimulación temprana para potenciar el desarrollo psicomotor en niños y niñas de 2 a 3 años de edad del área urbana y rural. Quito: ECE. Obtenido de http://200.12.169.19/handle/25000/7640

Martínez Ch, G., Guevara A, A., \& Valler O, M. (2016). El desempeño docente y la calidad educativa. RA Ximhay, 1234-134.

Navas, D. (2018). Desarrollo cognitivo, sensorail, motor y psicomotor en la infancia . México: IC Editorial.

Ochoa C, G. (2019). Relación entre la situación socio-económica familiar y el desarrollo motor grueso en niños de 5 a 18 meses que acuden a dos guarderías una del sector urbano y otra del sector rural de la cuidad de Quito: comparación mediante la escala motora infantil de A. Quito: PUCE.

Ortiz, N. (2005). Reflexiones sobre la Evaluacióndel Desarrollo Infantil en Proyectos Sociales. Cuaderno de la Red sobre la Primera Infancia. Desarrollo Infantil Escalas e Indicadores, 4, 10-33. Obtenido de http://www.gc-al.org.co.

Pacheco, G. (2019). Psicomotricidad en educación inicial. Quito: CEMEI. Obtenido de https://www.academia.edu/download/56093758/psicomotricidad_nivel_inicial.p df

Poblete , F., Toro, P., \& Cruzat, E. (2016). Desarrollo motor grueso en escolares de zona urbana y rural. Revista Horizonte Ciencias de la Actividad Física, 7(1), 59-66. Obtenido de http://revistahorizonte.ulagos.cl/index.php/horizonte/article/view/73

Reboiras , A., Cancela M, J., \& Ayán , C. (2015). Valoración de la competencia motriz en niños y niñas de Educación Infantil a través de la batería MOT 4-6. Journal of sport and health reserch, 7(2), 127-138.

Reyes A, I., Vaca C, E., \& Panchi, E. (2015). Aplicación de instrumentos lúdicos para el desarrollo de la motricidad fina de niños y niñas de 4 años de edad con variables de género, clase, etnicidad y territorial, incluye uso de Tic's. Revistya Anales, 1(373), 311-327. Obtenido de http://200.12.169.32/index.php/anales/article/view/1353

Rodríguez I, C. (2015). Cristina. Evaluación de las habilidades básicas: motriz y lenguaje en los niños y niñas de 1 a 3 años de los centros infantiles del buen vivir 
urbano y rural del cantón Cuenca. Cuenca: Universidad del Azuay. Obtenido de http://201.159.222.99/handle/datos/4013

Roque I, J., Otero L, F., Burgués L, P., \& Otegi E, J. (2018). Emorregulación y pedagogía de las conductas motrices. Acciónmotriz(21), 67-76. Obtenido de https://dialnet.unirioja.es/servlet/articulo?codigo $=6597291$

Rosero V, E. (2016). Estudio Comparativo del Desarrollo Psicomotor en Niños/As de 1 A 3 años del Centro Infantil del Buen Vivir "Centro Pucara" y "Gotitas De Amor" del Cantón Antonio Ante de la Provincia De Imbabura. La U Investiga, 3(3), 3850 .

Obtenido

de

http://revistasojs.utn.edu.ec/index.php/lauinvestiga/article/view/235

Salazar C, P., \& Jiménez D, J. (2020). Diferencias en la competencia motriz percibida de una población infantil según la región geográfica. Sportis, 6(2), 246-265. Obtenido de https://ruc.udc.es/dspace/handle/2183/25532

Senescyt. (2019). Información Estadística sobre Educación Superior, Ciencia, Tecnología e Innovación. Obtenido de Estadisticas : https://www.educacionsuperior.gob.ec/informacion-estadistica-sobre-educacionsuperior-ciencia-tecnologia-e-innovacion/

Silva Martínez, G. (2018). Desarrollo integral de niños de 4 a 5 años de una escuela rural vs. escuela urbana. Quito: PUCE. Obtenido de http://repositorio.puce.edu.ec/handle/22000/16096

Zuta T, R., \& Comeca P, S. (2019). La psicomotricidad en niños de tres y cuatro años en instituciones educativas, zona urbana y periférica, Luy a Lamud,. Chachapoyas: Universidad Nacional de Toribio Rodriguez de Mendoza de Amazonas. Obtenido de http://repositorio.untrm.edu.pe/handle/UNTRM/1929

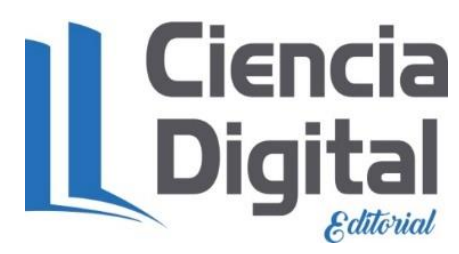




\section{PARA CITAR EL ARTÍCULO INDEXADO.}

Colcha Concha, J. P., \& San Lucas Solórzano, C. E. (2021). Análisis comparativo del desarrollo motriz de niños de 4 y 5 años en contextos educativos urbanos y rurales. Explorador Digital, 5(3), 61-84. https://doi.org/10.33262/exploradordigital.v5i3.1752

\section{¿Ciencia}

El artículo que se publica es de exclusiva responsabilidad de los autores y no necesariamente reflejan el pensamiento de la Revista Explorador Digital.

El artículo queda en propiedad de la revista y, por tanto, su publicación parcial y/o total en otro medio tiene que ser autorizado por el director de la Revista Explorador Digital.
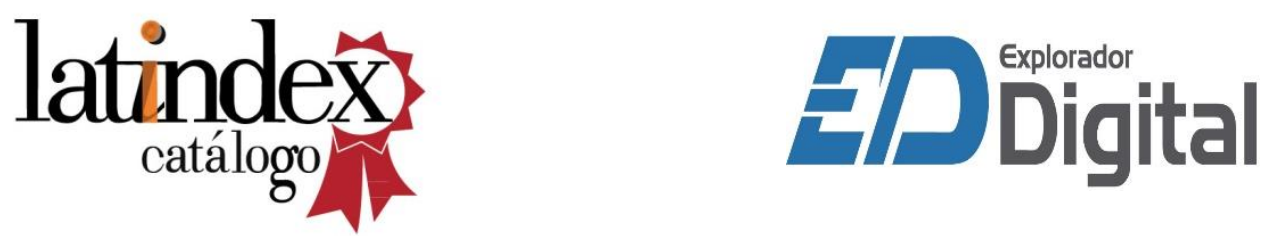